\title{
Surgical Management of a Functional Paraganglioma of the Infratemporal Fossa
}

\author{
Andrew G. Tritter ${ }^{1,2}$ Jesse Selber ${ }^{3}$ Michael E. Kupferman ${ }^{1}$ \\ ${ }^{1}$ Department of Head and Neck Surgery, The University of Texas MD \\ Anderson Cancer Center, Houston, Texas, United States \\ 2 Baylor College of Medicine, Houston, Texas, United States \\ ${ }^{3}$ Department of Plastic Surgery, The University of Texas MD Anderson \\ Cancer Center, Houston, Texas, United States \\ Address for correspondence Michael E. Kupferman, MD, FACS, \\ Associate Professor, Department of Head and Neck Surgery, MD \\ Anderson Cancer Center, 1515 Holcombe Blvd, Unit 1445, Houston, TX \\ 77030, United States (e-mail: MEKupfer@mdanderson.org). \\ J Neurol Surg Rep 2015;76:e1-e7.
}

\begin{abstract}
Keywords

- functional paraganglioma

- infratemporal fossa

- maxillary swing

- glomus tumor

Background Paragangliomas are rare neural crest tumors that can manifest in the head and neck as either functional or more commonly as nonfunctional lesions. Paragangliomas of the infratemporal fossa are exceedingly rare, with no more than a handful of documented cases. Like other tumors of this space, surgical management is challenging on account of complex anatomy and nearby critical structures.

Methods A 44-year-old man presented with a right infratemporal fossa functional paraganglioma. Following preemptive embolization and autonomic pharmacotherapy, his tumor was successfully resected via a transfacial maxillary swing approach.

Results This case highlights the surgical management of a functional paraganglioma of the infratemporal fossa while demonstrating the effectiveness of a transfacial maxillary swing approach for both exposure and resection.

Conclusion Although this report summarizes much of the literature on paragangliomas, there is still much to uncover regarding the fundamental features and genetic etiology of these lesions.
\end{abstract}

\section{Introduction}

Paragangliomas (PGLs) are rare and highly vascular tumors of neural crest origin that comprise only $0.6 \%$ of all head and neck tumors. ${ }^{1}$ Formerly known as glomus tumors, or chemodectomas, PGLs develop from the extraadrenal chromaffin and chief cells of autonomic paraganglia in various locations throughout the body, usually in association with the walls of blood vessels or specific nerves. ${ }^{2,3}$ Although these generally benign lesions most often present by exerting local effects on surrounding structures, a smaller subset of tumors can retain their neuroendocrine functionality and present with signs and symptoms of excess circulating catecholamines. These hypersecretory or functional PGLs can further complicate treatment by requiring a significant amount of autonomic pharmacotherapy to decrease the hemodynamic risks associated with any physical manipulation of the tumor itself.

received

January 3, 2014 accepted after revision

May 7, 2014

published online

January 16, 2015
Of the various reported locations for PGL to develop in the head and neck, the carotid body has been cited as the most common site, followed in order of frequency by the jugulotympanic region, vagal body, and less common sites like the larynx, pharynx, and ciliary ganglion. ${ }^{1}$ The infratemporal fossa (ITF) represents an especially rare location for a PGL to emerge, with only a handful of cases in the literature describing such a presentation. ${ }^{4,5}$ Given the complex anatomy of the anterior skull base and the critical neurovascular structures within the ITF, successful resection of such a highly vascular tumor within this space presents numerous perioperative and intraoperative challenges. This report describes the management of a rare hypersecretory PGL of the infratemporal fossa.

\section{Case Report}

A 44-year-old white man was referred to the Head and Neck Surgery service for evaluation of a right skull base mass, after
License terms

Stuttgart · New York

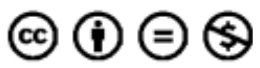




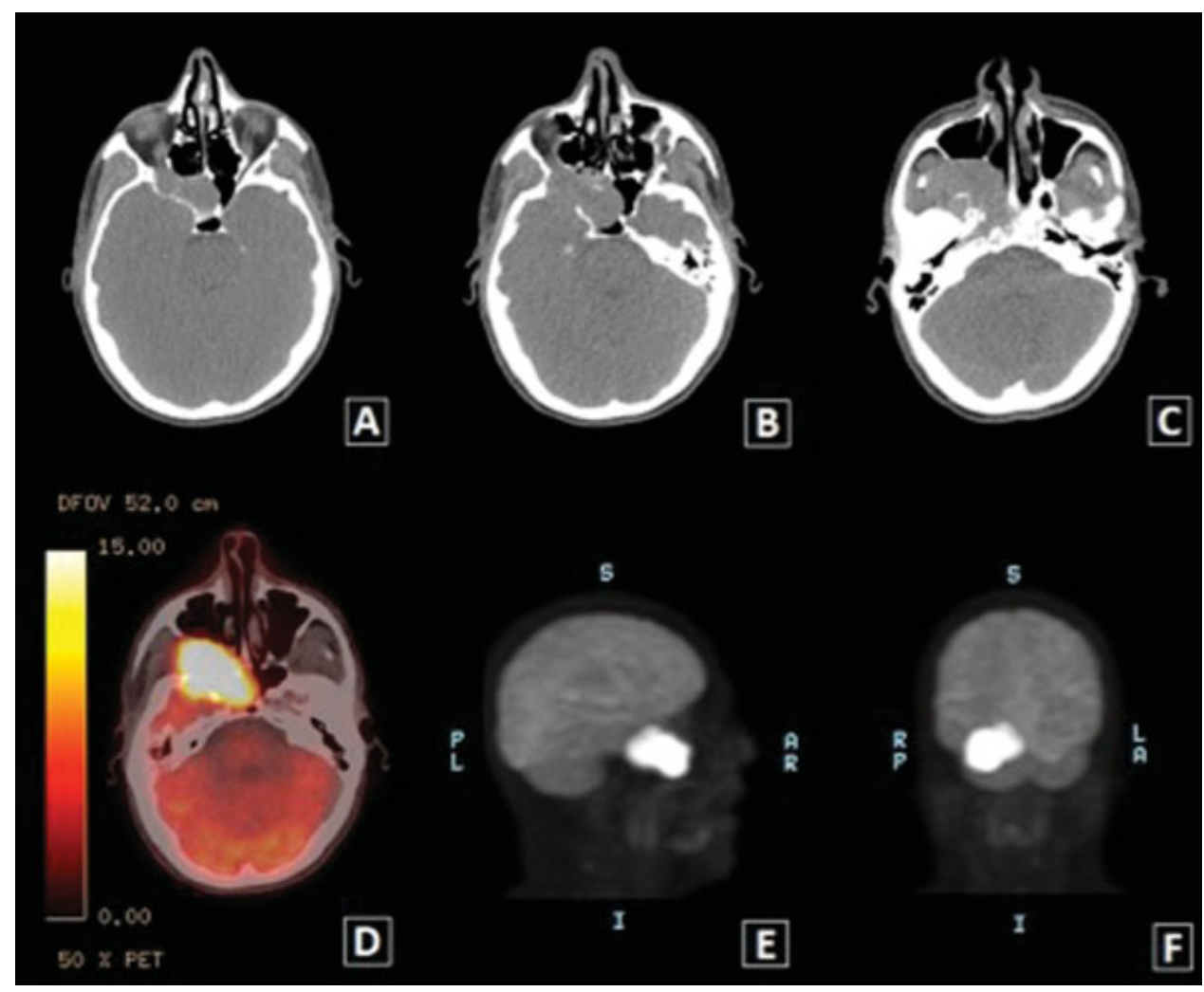

Fig. 1 Initial positron emission tomography (PET)/computed tomography imaging. (A-C) Superior to inferior progression demonstrating a soft tissue mass in the right infratemporal fossa with partial extension into the middle cranial fossa. (D) PET image showing intense focus of uptake from the lesion. (E, F) Sagittal and coronal images of enhancing lesion, inverted to show contrast.

initially presenting several months earlier to his primary physician for a 1-year history of hypertension, tachycardia, flushing, and diaphoresis. His initial work-up had revealed an elevated 24-hour urine normetanephrine and total metanephrine at $3122 \mu \mathrm{g}$ and $3223 \mu \mathrm{g}$, respectively. Diagnostic positron emission tomography/computed tomography imaging did not reveal any evidence of a pheochromocytoma, although a $6 \mathrm{~cm}$ metabolically active mass (standardized uptake value: 24.6 ) involving the ITF and pterygomaxillary space was identified (-Fig. 1). He was incidentally noted to

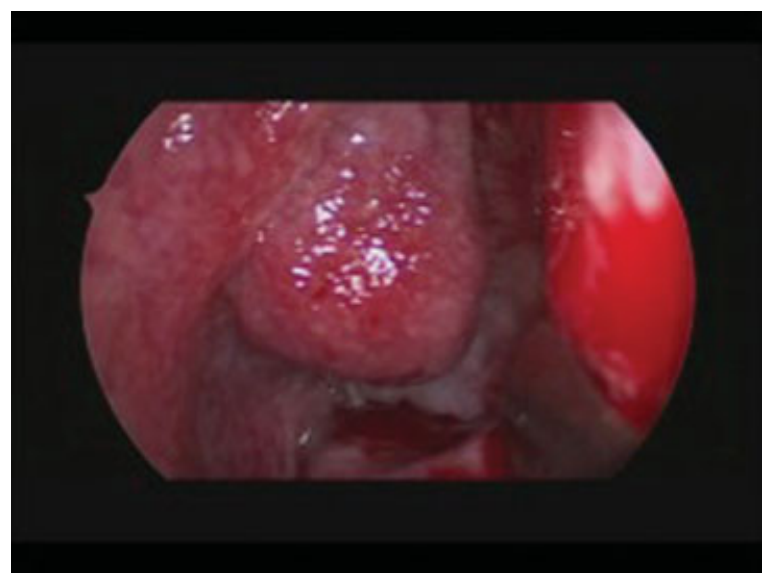

Fig. 2 Soft tissue mass emanating from the right middle meatus as seen on fiberoptic nasal endoscopy. have two metabolically inactive lytic lesions of the T10-T11 vertebrae that were later revealed to be multiple myeloma.

At the time of referral to our service, the patient complained of increasing right-sided nasal congestion, although he denied any nasal drainage, epistaxis, cranial neuropathies, or ocular issues. His head and neck examination was negative for any overt masses by palpation, although fiberoptic nasal endoscopy did reveal a hypervascular soft tissue mass emanating from the right middle meatus with involvement of the posterior aspect of the right maxillary sinus (-Fig. 2). Magnetic resonance imaging (MRI) of the skull base was acquired that demonstrated a large skull base mass bulging into the middle cranial fossa with numerous internal flow voids and associated destruction of the right sphenoid sinus, greater sphenoid wing, orbital fissures, and right pterygopalatine fossa (-Fig. 3). Additionally, the mass appeared to be intimately associated with the right optic fissure, optic canal, and cavernous carotid artery. These imaging findings were consistent with either a juvenile angiofibroma or a PGL. However, given the patient's endocrine findings, the lesion was diagnosed clinically as a functional PGL.

In light of the patient's uncontrolled blood pressure secondary to excess circulating catecholamines, he was started on phenoxybenzamine and metoprolol to achieve adequate $\alpha$ - and $\beta$-blockade, respectively. On the day prior to surgical resection he underwent an uncomplicated preemptive embolization of the lesion. Intraoperative surgical navigation was used for the identification of critical neurovascular 


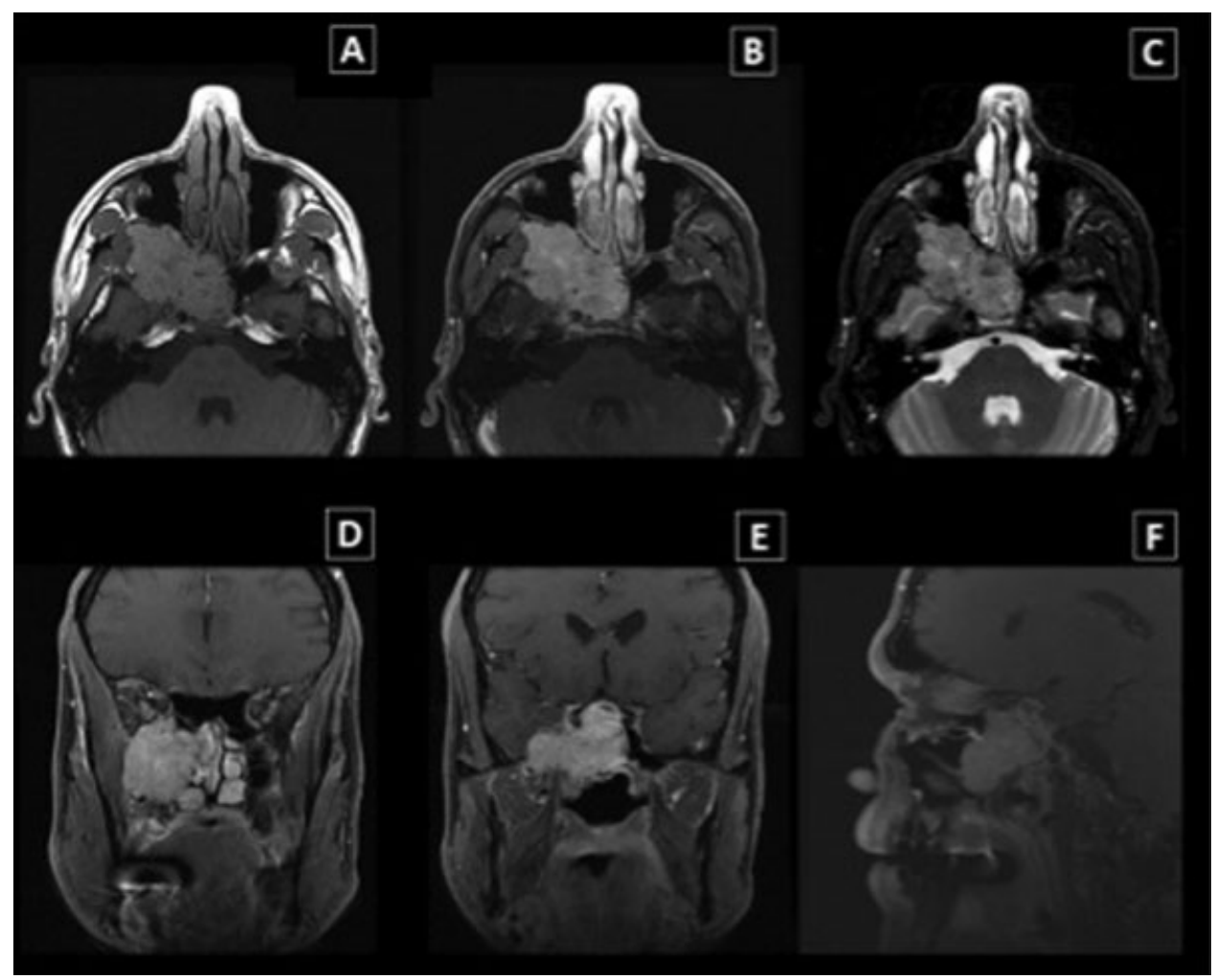

Fig. 3 Preoperative magnetic resonance Imaging of the head and face. (A) Axial T1 view of lesion in right infratemporal fossa. (B) Axial T1 view with contrast; same level as (A). (C) Axial T2 view; same level as (A). (D, E) Anterior to posterior sequence of coronal T1 images with contrast. (F) Sagittal T1 image with contrast.

structures. The surgical approach began with a right selective neck dissection (levels II-III), performed to obtain vascular control of the carotid artery and internal jugular vein, and to expose the lower cranial nerves. A Weber-Ferguson approach with a right maxillary swing was then utilized to access the tumor in the ITF. After osteotomizing the inferior strut, the medial orbital floor and rim were osteotomized medial to the infraorbital nerve, and the maxilla was swung laterally in continuity with the cheek flap ( - Fig. 4). Following dissection of the lesion off of the orbital floor and pterygopalatine fossa,

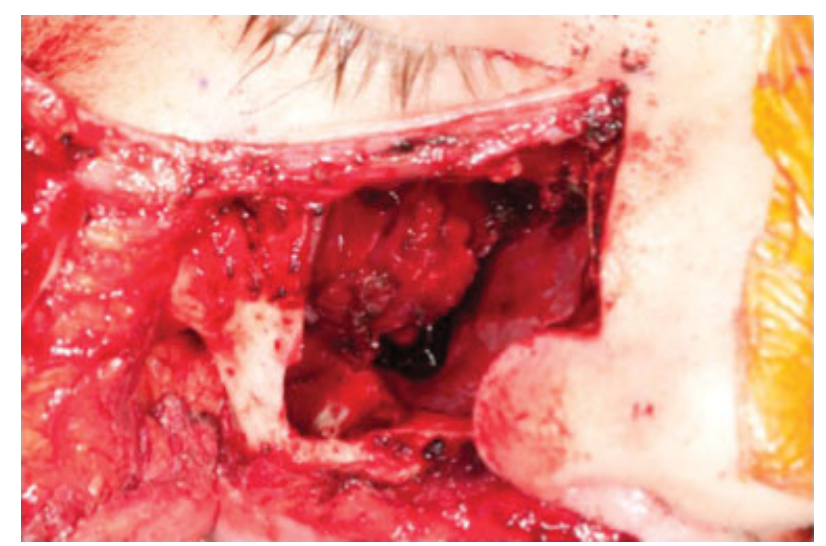

Fig. 4 Transfacial exposure of the patient's paraganglioma situated deep in the right infratemporal fossa. the specimen was mobilized medially, and the vidian and internal maxillary arteries were divided and ligated. These two arteries were observed to be the principal feeding vessels to the lesion without any significant contributions from the internal carotid artery or other major vessels, in concordance with the preoperative arteriogram. The tumor was then carefully dissected away from the sphenoid sinus and delivered off the undersurface of the middle cranial fossa, where the tumor had eroded the bone and was minimally adherent to the still-intact dura.

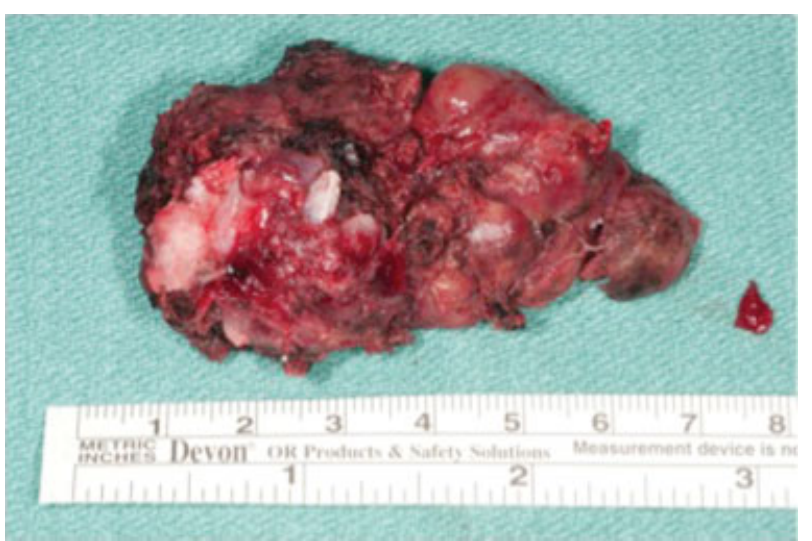

Fig. 5 En bloc resection of paraganglioma from the right infratemporal fossa. 


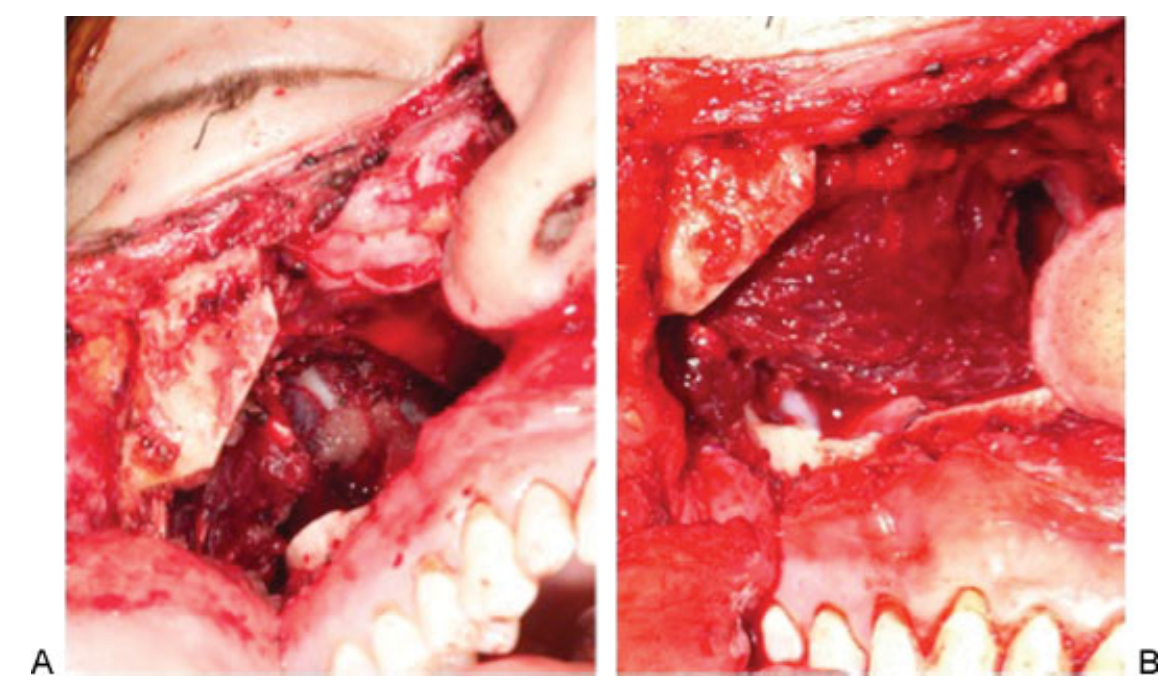

Fig. 6 (A) Evacuated infratemporal fossa following resection of tumor with portions of internal carotid artery, cavernous sinus, and anterior temporal lobe exposed. (B) Pedicled temporalis muscle flap covering the previously exposed defect.

The specimen was removed en bloc and sent to pathology, which confirmed the diagnosis of a PGL with negative surgical margins ( - Fig. 5). No metastatic nodes were identified in the neck dissection specimen. Hemostasis was adequately achieved, and a small dural tear was repaired with Gelfoam (Pfizer, New York, United States) and Tisseel (Baxter, Deerfield, Illinois, United States). Given the significant amount of bony erosion of the middle fossa caused by the tumor, as well as the transfacial exposure, a craniotomy was not required to improve visualization during the dural repair. The surgical defect was then reconstructed with a pedicled rotational temporalis muscle flap, which provided vascularized soft tissue coverage of the exposed internal carotid artery, cavernous sinus, and anterior temporal lobe ( - Fig. $\mathbf{6 A}, \mathbf{6 B}$ ). The maxilla was then repositioned in its anatomical location with titanium plates (-Fig. $\mathbf{7}$ ), and the skin and mucosal surfaces were closed in a layered fashion.

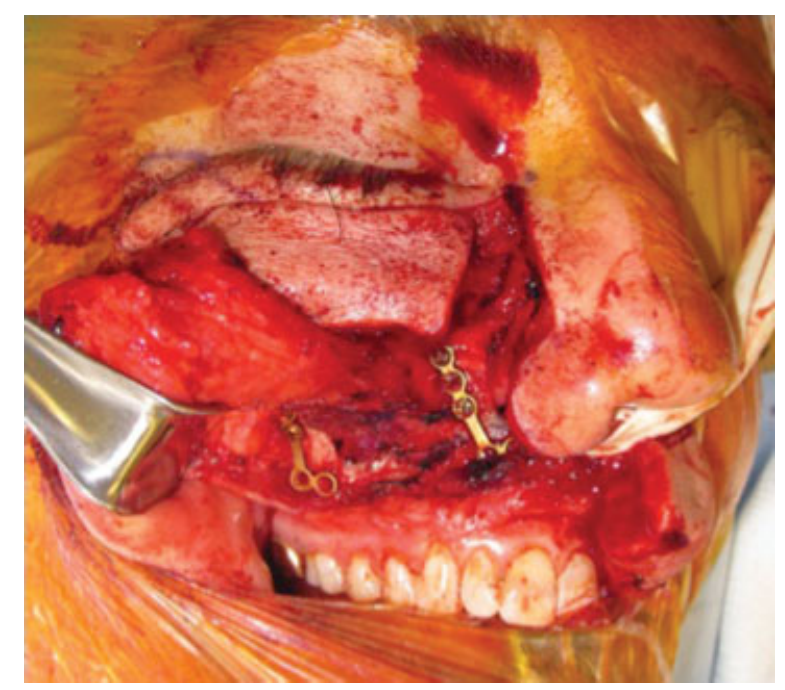

Fig. 7 Repositioning of the maxilla with titanium plating system prior to closure of surgical incisions.
In spite of having undergone preoperative embolization, the prominent vascularity of the tumor posed a significant challenge to the operation, and the overall estimated blood loss was 2 L. Nevertheless, there were no signs of hemodynamic instability during the course of the resection. He was extubated on postoperative day 1 , and was subsequently weaned off all antihypertensive medications during his hospital course.

The patient required partial debridement of the temporalis flap 10 weeks postoperatively for partial flap necrosis, which was performed via a right Caldwell-Luc, with endoscopic exploration of his right maxillary sinus. He also underwent endoscopic dacryocystorhinostomy with silicone tube placement for lacrimal duct obstruction. He initiated systemic therapy for his multiple myeloma 3 months after his initial surgical resection, and at 1-year follow-up is free of recurrence, with an excellent cosmetic and functional outcome. A selection of images from a follow-up MRI 9 months postoperatively is provided for comparison with the patient's initial imaging (-Fig. 8).

\section{Discussion}

Although tumors of the ITF are rare, they have long been a point of debate and discussion regarding the optimal surgical approach, due to the significant anatomical challenges they pose to the surgeon. Of the different types of tumors reported to arise primarily from the ITF, adenoid cystic carcinoma is regarded as the most common malignant neoplasm; peripheral nerve tumors account for most of the benign lesions. ${ }^{6}$ Regardless of the specific pathology, the deeply concealed location of this confined anatomical space with numerous important structures makes any approach to the ITF exceedingly difficult. Situated without a specific anatomical floor, the ITF is bounded medially by the lateral pterygoid plate, laterally by the mandibular ramus, anteriorly by the posterolateral aspect of the maxilla, posteriorly by the sphenoid spine and articular tubercle of the temporal bone, and superiorly by the greater wing of the sphenoid. ${ }^{7,8}$ Some of the critical 


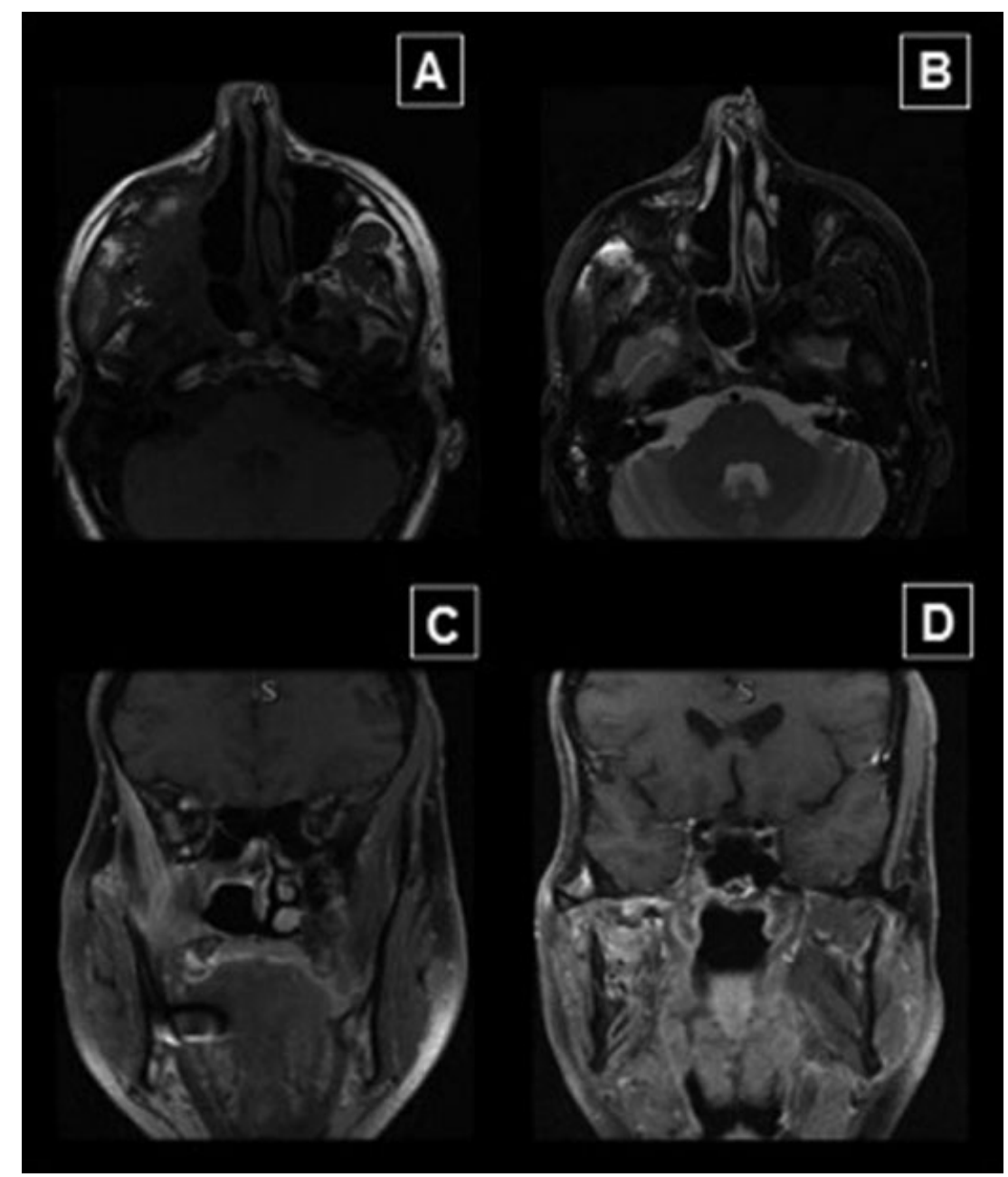

Fig. 8 A 10-month postoperative magnetic resonance imaging of the head and face. (A) Axial section T1. (B) Axial section T2 fluid-attenuated inversion recovery (FLAIR). (C, D) Coronal section T1 FLAIR with contrast.

structures encountered within this space include the internal maxillary artery, otic ganglion, chorda tympani, mandibular branches of the trigeminal nerve, and muscles of mastication like the lateral and medial pterygoids. ${ }^{8,9}$

The specific location of the tumor within this space, along with its relationship to nearby structures and extension into any adjacent compartments (e.g., the orbit, cranial vault, etc.), remain the primary determinants in the selection of the most appropriate surgical approach. It is this fact that has driven the development of multiple improved techniques for resection of ITF tumors over the past century. Building on the work of their peers, pioneers like Sewall in the 1920s, Conley, Barbosa, and Crockett in the 1960s, and Fisch in the 1970s helped lead the development of maximizing surgical exposure to the ITF. ${ }^{6}$ Their work, along with that of many others, has provided modern craniofacial surgery with a large variety of options suited to the resection of any number of skull base malignancies. According to Joo et al, these techniques can be grouped into two main categories: anterior and lateral (Isolan et al and Otremba et al have further suggested "inferior" as a third category to encompass the transmandibular and transcervical approaches). ${ }^{7,9}$ Among the lateral approaches, the Fisch type A, $\mathrm{B}, \mathrm{C}$, and $\mathrm{D}$ procedures are regarded as some of the most revolutionary for their ability to permit treatment of lesions involving the temporal bone and skull base. ${ }^{7}$ Variations on these procedures, such as the preauricular subtemporal approach described by Sekhar, have also been described. However, although these approaches do permit good exposure and vascular control, access to tumors extending medial to the pterygomaxillary fissure or across the midline is one of several recognized limitations to this approach., ${ }^{7,9}$

Among the anterior routes of access, the transmaxillary approach is one of the more common means of accessing the ITF, particularly for tumors extending to the nasopharynx. The natural space of the maxillary sinus makes the procedure fairly safe and simple; however, these approaches provide minimal exposure with relatively distal access, which can especially limit the resection of larger tumors and often require transfacial incisions. ${ }^{8,9}$ This same limitation also applies to endoscopic techniques of accessing the anterior skull base. Endoscopy has been used for resection of other vascular tumors, such as angiofibromas, but large lateral lesions with significant involvement of critical anatomical structures like the internal carotid artery and dura, as seen in our patient, are more safely accessed via the transfacial or infratemporal fossa approaches. 
Although many variations on these types of approaches have been extensively described in the literature, we used a variation of the maxillary swing, with preservation of the integrity of the hard palate. The maxillary swing is a fairly radical procedure whose usefulness was first demonstrated by Wei et al in 1991 as a means of resecting tumors in the nasopharyngeal and parapharyngeal space. ${ }^{8}$ There has been a fair amount of disagreement regarding the usefulness of the maxillary swing for resection of tumors in the ITF, but Otremba et al, as well as Honda et al, were able to describe its successful implementation in several cases of ITF tumors. ${ }^{8} 10$ Similar to our patient, this approach offers excellent exposure to the middle cranial fossa, particularly when tumors arise in the medial aspect of the skull base. For this case in particular, the highly vascular nature of the lesion necessitated exposure from a more direct and medial route, in anticipation of potential carotid and cavernous sinus dissection. Facial incisions are generally necessary, but the cosmetic outcomes are favorable when radiotherapy is not anticipated, and restoration of the midface bony framework is possible.

Head and neck PGLs generally present in the third or fourth decade of life as asymptomatic space-occupying lesions. ${ }^{11,12}$ They are most often benign tumors with a $10 \%$ incidence of malignancy. There is also a well-established familial association that has been observed in upward of $10 \%$ of PGLs, owing predominantly to mutations in three of the four succinate dehydrogenase subunit genes (SDHB, SDHC, and $S D H D){ }^{1,5}$ This genetic predisposition carries an increased risk for multifocal tumor development, with an $80 \%$ chance of multicentricity in familial cases as opposed to only a 10 to $20 \%$ chance in sporadic cases. ${ }^{1,5}$ The genetic characteristics of familial PGLs have been well described in various publications, such as Martin et al 2007 and Papaspyrou et al 2011, although $S D H D$ is the most commonly implicated gene in the development of benign familial PGLs of the head and neck, and SDHB mutations have the highest rate of developing malignant PGLs. ${ }^{5,12}$ Papaspyrou et al did report one case of their 175 patient series in which the patient was found to be positive for a mutation in the SDHC gene after presenting with multiple malignant PGLs of the ITF, carotid body, and jugulotympanic region..$^{5}$ Although this observation is certainly interesting, a large amount of additional data are needed before any specific conclusions on the topic can be made.

As a final comment from the standpoint of genetics, the simultaneous occurrence of both PGL and multiple myeloma in our patient presents a thought-provoking clinical coincidence. To our knowledge there is no known association between multiple myeloma and PGLs, or between multiple myeloma and the SDH gene family. A clinical indication for genetic testing in our patient has not yet arisen, but it would be interesting to further elucidate the genetic relationship between these pathologies.

As demonstrated by this case, functional PGL can present an additional challenge in management that is not commonly observed in other types of head and neck PGL. By virtue of their cellular origin, all PGLs possess cytoplasmic neurosecretory granules that provide them with the theoretical ability to manifest as functional lesions. Yet in spite of this fact, only 1 to $3 \%$ of head and neck cases actually present with physiologic signs of catecholamine excess. ${ }^{11}$ Interestingly, Kuhweide et al made the observation that those PGLs arising in the more medial aspects of the skull base (including the ITF) tended to be more prone to the production of biogenic amines, and by extension, more likely to be catecholamine secreting. Although this is a rare location for PGLs to arise in the first place, it has been speculated that the presence of specific parasympathetic paraganglia (the otic ganglion, specifically, in the case of ITF PGLs) is the underlying source of these tumors. ${ }^{3}$

Regardless of their etiology, functional PGLs require the administration of autonomic pharmacotherapy as part of their management. Once biochemical documentation of excess catecholamine secretion has been established, and surgical resection has been chosen as definitive treatment, combined $\alpha$ - and $\beta$-adrenergic blockades should be initiated preoperatively to control blood pressure and prevent intraoperative hypertensive crises. ${ }^{13}$ Young proposed that $\alpha$-adrenergic blockade be initiated at least 7 days before surgery, with the addition of $\beta$-adrenergic blockade once the former is adequately achieved (e.g., 3 days preoperatively). He reported the need for postoperative hemodynamic management in only $7 \%$ of patients undergoing resection of catecholaminesecreting tumors who had used this preoperative approach. ${ }^{13}$

In conjunction with preoperative pharmacotherapy, embolization has also been shown to provide some additional benefits in the management of certain functional PGLs. Preemptive embolization has been shown to decrease tumor size by up to $25 \%$ and decrease blood loss during surgical dissection among all head and neck PGLs, and it also provides the theoretical benefit of decreasing catecholamine production by starving the tumor of necessary precursors. ${ }^{2,3}$ More data are still needed on the outcomes of functional PGL resection with and without preemptive embolization, although one could speculate that embolization would further decrease the risk of intraoperative hypertensive crises that may occur from manipulation-induced release of catecholamines in those patients with insufficient adrenergic blockade. It should be noted, however, that the indications for embolization in the management of head and neck PGLs still remain somewhat disputed. ${ }^{2}$ There is clear evidence for its efficacy in certain circumstances, but the potential neurovascular consequences in the hands of an inadequately trained radiologist highlight the importance of weighing the risks of embolization against the projected benefit to the surgical team on a case-by-case basis.

A fair body of work has been dedicated to the role of radiation therapy (RT) in the treatment of both functional and nonfunctional PGL. Although no definitive guideline exists for when RT should be used in these cases, evidence suggests that RT is moderately effective in the management of predominantly slow-growing, nonfunctional PGLs. This benefit may not be equally extendable to functional PGLs because certain catecholamine-secreting chromaffin cells have been shown to survive exposure to RT. ${ }^{14}$ Furthermore, the local tissue effects of irradiation remain a significant issue that the surgeon must address prior to initiation of definitive therapy. In a region where identification of intraoperative landmarks is critical, any tissue deformation incurred from preoperative RT can 
greatly increase the difficulty of an already challenging surgical resection.

Finally, although surgical resection was an appropriate treatment option for our patient, there is very little discussion in the literature concerning nonoperative management of functional PGL in the poor surgical candidate. As discussed by Patetsios et al in 2002 and Marzo et al in 1997 with regard to both functional and nonfunctional PGL of the head and neck, radiographic observation with or without the use of $\mathrm{RT}$ is the treatment mainstay for the elderly or severely infirm. ${ }^{15,16}$ Embolization and long-term therapeutic use of $\alpha / \beta$-blockers would seem to be a legitimate method of symptomatic treatment in nonsurgical candidates with functional PGL, but little evidence is available to support this strategy outside of preparation for surgical excision.

\section{Conclusion}

This case demonstrates several of the significant challenges in approaching the ITF for the surgical resection of lesions in this space. Moreover, the perioperative and intraoperative management of a functional PGL of the ITF was discussed. The successful and complete removal of our patient's tumor serves to demonstrate the effectiveness of a transfacial maxillary swing approach for exposure and resection of a central skull base lesion with extension into the middle cranial fossa. The advantages and disadvantages of varied approaches should be familiar to the surgeon approaching this anatomical region. The rarity of features surrounding this case highlights the fact that there is still much to uncover regarding the fundamental features and genetic etiology of these lesions.

\section{References}

1 Lee JH, Barich F, Karnell LH, et al; American College of Surgeons Commission on Cancer American Cancer Society. National Cancer
Data Base report on malignant paragangliomas of the head and neck. Cancer 2002;94(3):730-737

2 Persky MS, Setton A, Niimi Y, Hartman J, Frank D, Berenstein A. Combined endovascular and surgical treatment of head and neck paragangliomas-a team approach. Head Neck 2002;24(5): 423-431

3 Kuhweide R, Lanser MJ, Fisch U. Catecholamine-secreting paragangliomas at the skull base. Skull Base Surg 1996;6(1):35-45

4 Cantrell RW, Kaplan MJ, Atuk NO, Winn HR, Jahrsdoerfer RA. Catecholamine-secreting infratemporal fossa paraganglioma. Ann Otol Rhinol Laryngol 1984;93(6 Pt 1):583-588

5 Papaspyrou K, Mewes T, Rossmann H, et al. Head and neck paragangliomas: Report of 175 patients (1989-2010). Head Neck 2012; 34(5):632-637

6 Tiwari R, Quak J, Egeler S, et al. Tumors of the infratemporal fossa. Skull Base Surg 2000;10(1):1-9

7 Isolan GR, Rowe R, Al-Mefty O. Microanatomy and surgical approaches to the infratemporal fossa: an anaglyphic threedimensional stereoscopic printing study. Skull Base 2007;17(5): 285-302

8 Otremba M, Adam S, Omay SB, Lowlicht R, Bulsara KR, Judson B. Maxillary swing approach for extended infratemporal fossa tumors. Laryngoscope 2013;123(7):1607-1611

9 Joo W, Funaki T, Yoshioka F, Rhoton ALJr. Microsurgical anatomy of the infratemporal fossa. Clin Anat 2013;26(4):455-469

10 Honda K, Asato R, Tanaka S, Endo T, Nishimura K, Ito J. Vidian nerve schwannoma with middle cranial fossa extension resected via a maxillary swing approach. Head Neck 2008;30(10):1389-1393

11 Pellitteri PK, Rinaldo A, Myssiorek D, et al. Paragangliomas of the head and neck. Oral Oncol 2004;40(6):563-575

12 Martin TP, Irving RM, Maher ER. The genetics of paragangliomas: a review. Clin Otolaryngol 2007;32(1):7-11

13 Young WF Jr. Paragangliomas: clinical overview. Ann N Y Acad Sci 2006;1073:21-29

14 Schwaber MK, Gussack GS, Kirkpatrick W. The role of radiation therapy in the management of catecholamine-secreting glomus tumors. Otolaryngol Head Neck Surg 1988;98(2):150-154

15 Patetsios P, Gable DR, Garrett WV, et al. Management of carotid body paragangliomas and review of a 30-year experience. Ann Vasc Surg 2002;16(3):331-338

16 Marzo SJ, Leonetti JP. Current management of glomus jugulare tumors. Neurosurg Q 1997;7(2):103-109 